\title{
Determination of the friction coefficients of chestnut (Castanea sativa Mill.) sawn timber**
}

\author{
José Ramón Villar-García*, Pablo Vidal-López, Antonio J. Corbacho, and Manuel Moya \\ Department of Forest and Agricultural Engineering, University of Extremadura, Spain
}

Received February 21, 2019; accepted September 11, 2019

\begin{abstract}
This work provides the values of both the static and the kinetic friction coefficients for chestnut (Castanea sativa Mill.) of Spanish origin. Knowledge concerning these coefficients has its main application in the study of agroindustrial structures and machinery with timber members in contact. This determination was developed taking into account the timber anisotropy to establish surfaces and directions of slipping. A modified direct shear test device was used to conduct the tests and reproduce the tribological system. This procedure was functional and reliable and considered suitable for standardizing the friction measurement between timber surfaces, since this device is widely distributed in geotechnical and materials laboratories and the European codes do not specify a procedure or device to carry it out. The average values obtained were 0.46 for the static coefficient and 0.33 for the kinetic one, without considering the surfaces and directions of slipping. These values ranged between 0.36 and 0.55 for the static friction coefficient and between 0.28 and 0.39 for the kinetic friction coefficient depending on the direction considered and also taking into account the anisotropy of the timber. A good correlation was obtained between both coefficients, thus allowing for the estimation of the kinetic coefficient from the static one.
\end{abstract}

Keyw ords: wood, mechanical properties, friction coefficient, direct shear test

\section{INTRODUCTION}

Wood is a sustainable material with a significant carbon storage capacity, and timber products have a low-carbon footprint in their manufacture and use. They are widely used in agriculture, ranging from greenhouses, agroindustrial constructions and farm buildings to agricultural machinery;

\footnotetext{
*Corresponding author e-mail: jrvillar@unex.es

**This work was partially supported from the Junta de Extremadura (Spain) through Grants No. GR18175 and GR18193 (partially financed by European Regional Development Fund (ERDF), 2014-2020).
}

wood is of particular use in general construction. Friction between timber pieces is an important consideration in many of these uses, with examples ranging from a simple support in a wooden sleeper to the friction between the planks of a stress-laminated deck plate, or the force transmission in joints between timber members. The presence of friction is also significant in timber couplings, pulleys or bearings for conveyor belts, rolling mills and other kinetic systems in agroindustrial facilities and farm equipment. Several works (Villar et al., 2007, 2008, 2018, 2019; Koch et al., 2013; Aira et al., 2016) show the importance of knowing these coefficients in order to obtain both a deeper knowledge of the load transmission mechanisms and a suitable numerical simulation in timber joints. This is because load transmission is partially caused by friction forces, especially in the case of carpentry joints.

This friction force opposes the initiation of sliding or, once produced, the relative movement between two surfaces. At the molecular level, the interaction responsible for this force is the electromagnetic force between atoms and molecules, which must be overcome in order to produce slippage. In addition, irregularities and surface roughness must be taken into account. At this level, the irregularities or asperities deform both elastically and plastically, thereby producing an embedding in such a way that coupling between the highest points of a surface and the lowest ones of the opposite one oppose the slippage. Once slippage occurs, these asperities may be lifted, cut, torn or even melted due to the high temperatures that may be reached

(C) 2020 Institute of Agrophysics, Polish Academy of Sciences 
in certain cases, thus initiating the wear of the contacts between surfaces. Many microscopic particles are involved and their complex interaction is still not well understood, which makes the study of this phenomenon difficult. Therefore, for the usual engineering purposes, research works are usually conducted considering macroscopic behaviour alone, i.e. an experimental contact forces study.

Consider a solid block simply supported on a surface, its weight $\mathrm{W}$ exerts a contact force normal to the surface, $N$, of the same value, collinear, and in the opposite direction. If a horizontal force $\mathrm{P}$ is applied, a horizontal component $\mathrm{F}$ appears as a force reaction, which is the resultant of all contact forces produced between the block and the surface (Fig. 1). Force $\mathrm{N}$ is the integration of a force system distributed throughout the surface acting on the centroid of such a distribution. In this case, $\mathrm{N}$ does not have to be collinear with $\mathrm{W}$ and its location depends on the momentum balance of the solid. Whilst the block remains static, $\mathrm{P}$ is balanced by $\mathrm{F}$. When $\mathrm{P}$ is progressively increased, $\mathrm{F}$ will also be increased up to a maximum value $F_{\max }$, which corresponds to the point or condition of "imminent slipping" (Fig. 2). Once this point is surpassed, the friction force cannot balance the $\mathrm{P}$ value and slippage takes place. In that moment, and theoretically instantaneously, the $\mathrm{F}$ value decreases by about $20-25 \%$ for most materials. At this point, the sliding force is the kinetic friction force $\left(F_{k}\right)$ in contrast to the force exerted at rest or the static friction force $\left(F_{s}\right)$. If slippage takes place at a constant velocity, the force applied is equal to $F_{k}$. This behaviour at a macroscopic level is reproduced in the tests carried out in this work.

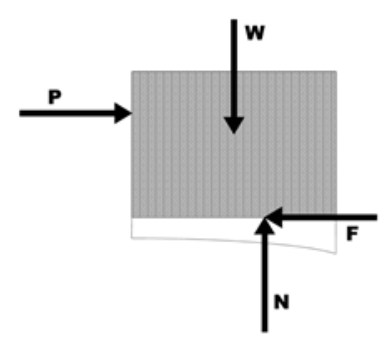

Fig. 1. Friction forces system on a rigid body.

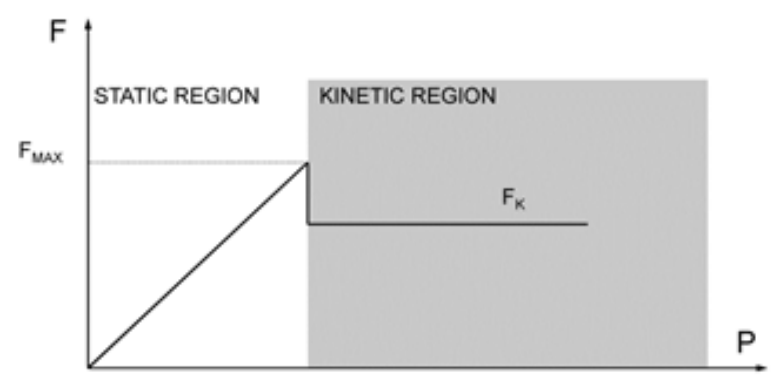

Fig. 2. Representation of the friction force F against the applied force $P$.
The solids mechanics considers with a great approximation that a direct proportionality exists between $F_{s}$ and $N$ (Tipler, 2008; Serway and Jewett, 2013), where the constant of proportionality is referred to as the static friction coefficient $\left(\mu_{s}\right)$ :

$$
F_{s}=\mu_{s} N .
$$

In a similar way, a proportionality exists between $F_{k}$ and $N$ by means of the kinetic friction coefficient $\left(\mu_{k}\right)$ :

$$
F_{k}=\mu_{k} N .
$$

Both coefficients are practically independent, for macroscopic purposes, of the size of the contact surface and the value of the normal force $N$ (Keller et al., 1993; Tipler, 2008; Serway and Jewett, 2013; Young and Freedman, 2016). In addition, the kinetic friction coefficient is independent of the velocity at small values (ranging between $1 \mathrm{~cm} \mathrm{~s}^{-1}$ and several m s${ }^{-1}$ (Tipler, 2008)) and slowly decreases as the velocity increases (Keller et al., 1993). Sometimes a stick-slip behaviour may be observed, a cycling (decrease and subsequent increase) in the friction force as sliding proceeds (ASTM G-115-10, 2018). The main factors this coefficient depends on are the nature and the conditions of both surfaces. A decrease in the kinetic friction force with respect to the static one reveals that the kinetic friction coefficient is, as a general rule, smaller than the static one for a wide range of materials. In the case of timber surfaces, these coefficients are affected by the moisture content, the roughness of the surface, and the surface characteristics (USDA Forest Products Laboratory, 2010). The last two factors are directly related to the anatomical properties, the timber stiffness, and the machining or treatment applied to the surfaces of the piece. As for the anatomical properties, it is because of the material anisotropy. The anisotropy refers to the orientation of the studied surface with respect to the orthotropic directions, longitudinal or parallel to the grain (also referred to as fibre), and perpendicular to the fibre, with both the radial and tangential directions, which originates different roughnesses for the same piece. Generally, the studies are conducted for orthogonal cuts to these orthotropic directions, since, in many cases, they correspond to the contact surfaces originated with the use of timber.

The machining or contact surfaces treatment will also affect the roughness. Hence, the experimental studies have to reproduce the best possible finish of the surfaces of interest (ASTM G115-10, 2018). In this work, the friction between sawn timber pieces is considered. With respect to humidity, for most timber species, the friction coefficients increase with moisture content, from ovendry until fibre saturation is reached. On the other hand, for moisture content under $20 \%$, the kinetic friction coefficients do not vary significantly with the slipping velocity, whereas for high moisture contents, the kinetic friction coefficients sharply decrease as velocity increases (USDA Forest Products Laboratory, 2010). 
In the European Standards, the only reference is the static coefficient, which can be found in the Eurocode 5-Part 2: Bridges (CEN EN 1995-2:2016, 2016). In this Standard, these values are provided for application in stress-laminated deck plates, which would correspond with conservative values, considering that they were conceived as calculation values, and just for softwoods (Table 1).

For timber, publications of general physics provide a value of 0.20 for the kinetic friction coefficient, whereas for the static coefficient it ranges between 0.25 to 0.50 (Serway and Jewett, 2013). In the case of more specific publications related to timber, Argüelles et al. (2013) provide generic values for dry timber ranging from between 0.25 and 0.70 for the static friction coefficient, and 0.15 to 0.40 for the kinetic one. In addition, the Wood Handbook (USDA Forest Products Laboratory, 2010) suggests, in a generic way, that the kinetic friction coefficients for smooth and dry timber $v s$. smooth and hard surfaces can range between 0.30 and 0.50 ; however, for an intermediate value of the moisture content, this coefficient ranges between 0.50 to 0.70 , whereas close to the fibre saturation point, it ranges between 0.70 and 0.90 . McKenzie et al. (1968) developed a device for studying the kinetic friction produced between timber and steel. The results produced by the device suggested a kinetic friction coefficient value of 0.45 for some timber to timber tests conducted, and a value of 0.60 for the static one, without specifying directions. Other values, which take into account the orthotropicity of timber, are reported by Kollman (1959), and are listed in Table 2.

The values corresponding to specific friction surfaces and directions were normally developed for use in the number simulation modelling of timber joints. Thus, Crespo et al. (2011) studied the friction coefficients for transverse surfaces (cuts perpendicular to the grain) in glued lamina-

Table 1. Calculation values for the static coefficient. Values taken from the Eurocode 5 (CEN EN 1995-2:2016, 2016)

\begin{tabular}{lllll}
\hline $\begin{array}{l}\text { Surface } \\
\text { orientation }\end{array}$ & \multicolumn{2}{c}{$\begin{array}{c}\text { Perpendicular } \\
\text { to the grain }\end{array}$} & \multicolumn{2}{c}{$\begin{array}{c}\text { Parallel } \\
\text { to the grain }\end{array}$} \\
\hline $\begin{array}{l}\text { MC }(\%) \\
\begin{array}{l}\text { Sawn timber- } \\
\text { Sawn timber }\end{array}\end{array}$ & 0.3 & 0.45 & 0.23 & 0.35 \\
$\begin{array}{l}\text { Planed timber- } \\
\text { Planed timber }\end{array}$ & 0.2 & 0.4 & 0.17 & 0.3 \\
$\begin{array}{l}\text { Sawn timber- } \\
\text { Planed timber }\end{array}$ & 0.3 & 0.45 & 0.23 & 0.35 \\
\hline
\end{tabular}

Table 2. Values of the friction coefficients for oak static $\left(\mu_{s}\right)$ and kinetic $\left(\mu_{k}\right)$ coefficients (Kollmann, 1959)

\begin{tabular}{llcc}
\hline Material & Surface orientation & $\mu_{s}$ & $\mu_{k}$ \\
\hline \multirow{3}{*}{ Oak } & Parallel to the grain & 0.62 & 0.48 \\
& Perpendicular to the grain & 0.54 & 0.34 \\
& Parallel - perpendicular & 0.43 & 0.19 \\
\hline
\end{tabular}

ted (glulam) timber specimens of Picea abies. L. Karst, obtained a value of 0.467 for the static friction coefficient and 0.310 for the average kinetic friction coefficient at a moisture content of $12 \%$. Soilán et al. (2011) studied glulam specimens of the same species, reducing the moisture content to $10 \%$ in order to obtain the coefficients in dovetail joint flanks. In this case, the values obtained were 0.42 for the static friction coefficient and 0.27 for the kinetic one. Aira et al. (2014) also studied radial surfaces and the sliding parallel to the grain for Scots pine, obtaining a value of 0.12 for $\mu_{s}$ and 0.08 for $\mathrm{m}_{\mathrm{k}}$, and values of 0.24 for $\mu_{s}$ and 0.17 for $\mu_{k}$, for transverse surfaces with a tangential sliding direction. Finally, Koch et al. (2013) stated that the range of values for the static friction of dry spruce ranged between 0.3 and 0.8 , while it ranged between 0.3 to 0.6 for the kinetic friction coefficient. When this author studied a tapered tenon joint, he considered a value of 0.45 for the static coefficient of friction in cuts perpendicular to the fibre against cuts at $45^{\circ}$ to the fibre, and 0.4 for cuts at $45^{\circ}$ with the grain frictioning against cuts parallel to the fibre.

From the literature review conducted herein, it may be concluded that, in many cases, sufficient data currently exists for timber in general, without distinguishing between different species; rarely do the coefficients refer to the different surfaces originating in the orthotropicity of the material. At the same time, no relevant studies have been found for chestnut timber, which is widely used not only in carpentry and coatings but also in agricultural facilities and general construction; especially in sawn timber in either modern realizations or in old ones where maintenance and rehabilitation are very important. In many cases, carpentry joints are used for sawn timber and its use is increasing due to computer-aided manufacturing. In addition, the use of hardwoods is currently increasing for structural purposes throughout Europe and insufficient research has been conducted in this regard. For all of these reasons, the study of the friction behaviour of chestnut timber (Castanea sativa Mill.) is considered to be of great interest.

\section{MATERIALS AND METHODS}

Current European codes do not include any standardized method for carrying out friction tests, in particular for wooden elements. The frame of reference could be the American Standard ASTM G-115-10 (2018), which includes recommendations for friction tests regarding the sample preparation, surfaces, and test method. In a generic way, this Standard states that the data that is required to be extracted with the test must be reproduced as faithfully as possible. Following this example, other researchers have developed specific devices designed to test the friction between timber specimens (Bejo et al., 2000; Crespo et al., 2011; Aira et al., 2014). However, the development of unique apparatus hampers the possibility of replicating the test conditions in other laboratories. Hence, in this work, 
a modified test device is used, the direct shear apparatus, it was initially developed in geotechnical laboratories but is also used in other applications, including the study of the mechanical properties of granular agricultural materials (Molenda et al., 2006). Hence, this device is proposed for obtaining the friction coefficients between the surfaces of solids, in this case, timber specimens. The procedure proposed here could help to standardize the test for obtaining friction coefficients.

The standard device of the Direct Shear Apparatus includes a shear box which is divided into two parts; the lower and the upper half-shear boxes where the soil or the granular material samples are placed. The shear box is placed in a box holder (or carriage) and the horizontal displacement of the carriage allows the shear tests to take place. The sample is sheared through a relative horizontal movement when the lower half-shear box slides and the displacement of the upper half-shear box is constrained. The force needed to constrain that movement is measured $(F)$. The vertical load is applied through a counter-balance device and is uniformly distributed along the sample with a loading plate.

The modification of the shear box in the direct shear apparatus for the study of friction has already been used previously by the authors, as in Moya et al. (2013) to determine the grain-to-wall friction coefficient for granular agricultural materials against concrete and steel surfaces. In order to implement the friction test between timber elements, it is necessary to arrange the specimens with the surfaces to be tested in such a way that there can be relative movement between them. The following modifications should be conducted to implement this procedure in such a way that the configuration shown in Figs 3 and 4 is reached: the shear box is completely removed, leaving the box holder completely empty; several rigid separators are placed on the box holder to allow the lower specimen to be placed within the centre of the carriage in order to maintain an adequate separation from the ends; the bridge of the shear box is attached to the connecting rod of the load cell, in such a way that the constraining force $(F)$ is transmitted to the load cell; the bridge must be in perfect contact with the upper specimen; a loading plate applies a vertical load (W) to the top surface of the upper specimen, the loading plate has dimensions such that the vertical load (W) applies a uniformly distributed load to the upper test specimen. With this arrangement, the lower specimen may be pushed by means of a horizontal actuator along the axis of the carriage where the lower specimen is placed. The horizontal displacement of the carriage is measured using an LVDT displacement sensor. The load cell has a capacity of $5 \mathrm{kN}$, which is adequate to measure the maximum force achieved during testing. The vertical load (W) is known and may be selected by means of the weights placed in the counter-balance device. A load of $2.5 \mathrm{kN}$ was applied, which is the same value as that used in friction tests conducted

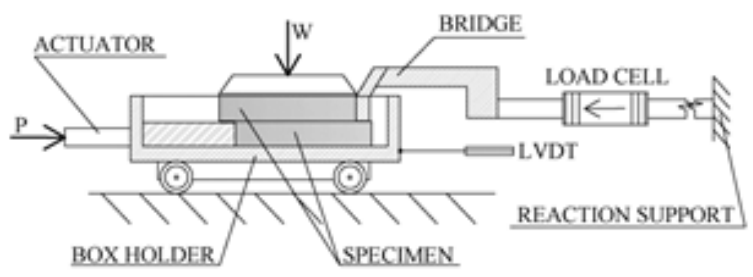

Fig. 3. Arrangement of the device for testing.

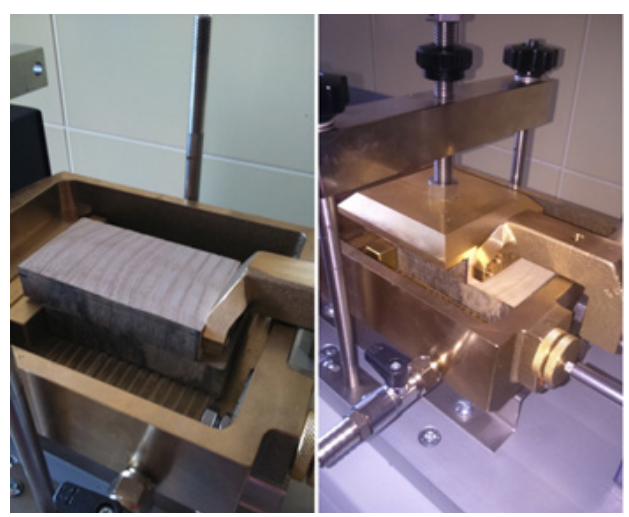

Fig. 4. Arrangement of the specimens for testing (left) and test performance (right).

by other authors (Crespo et al., 2011; Aira et al., 2014). The arrangement of the specimens should be such that the upper specimen is recessed by $1 \mathrm{~cm}$ thus avoiding the noise caused by the friction between the corners of the surfaces when the displacement begins (Aira et al., 2014), as shown in Figs 3 and 4. The test is conducted at a constant velocity, thus avoiding the appearance of inertial forces during displacement. That way, the force registered by the load cell, equals the force of kinetic friction $F_{k}$. The test velocity was $8 \mathrm{~mm} \mathrm{~min}^{-1}$, which was similar to that used in other friction tests (Crespo et al., 2011; Aira et al., 2014). The configuration of the direct shear device and the data acquisition software is such that the friction between other system elements does not affect the data registered for obtaining the friction coefficients. The software constantly collects data from the load cell and the displacement sensor LDVT, thus allowing for the determination of the friction coefficient at all times this data is later plotted to obtain $\mu_{s}$ and $\mu_{k}$. The first value is the maximum friction coefficient value corresponding to the point of the "imminent slip condition", whereas the second one reflects the moment when the friction coefficient is stabilized, keeping its value constant and tending to a horizontal asymptote.

In this work, chestnut timber (Castanea sativa Mill.) from various regions of Spain was used. For each test, the specimen is divided into two parts of equal dimensions: $105 \times 25 \times 50 \mathrm{~mm}$. These dimensions allow for an adequate fitting of the specimens within the modified device. In addition, it allows for a friction path of $20 \mathrm{~mm}$, which has been 


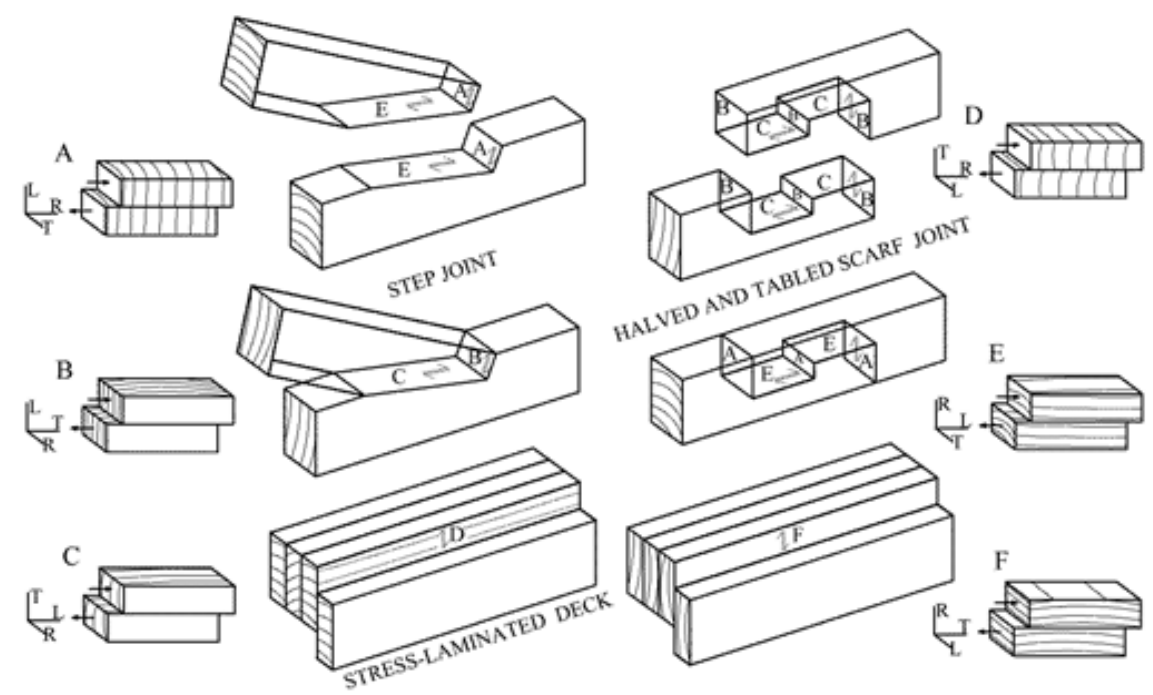

Fig. 5. Contact surfaces indicating the friction direction and some applications.

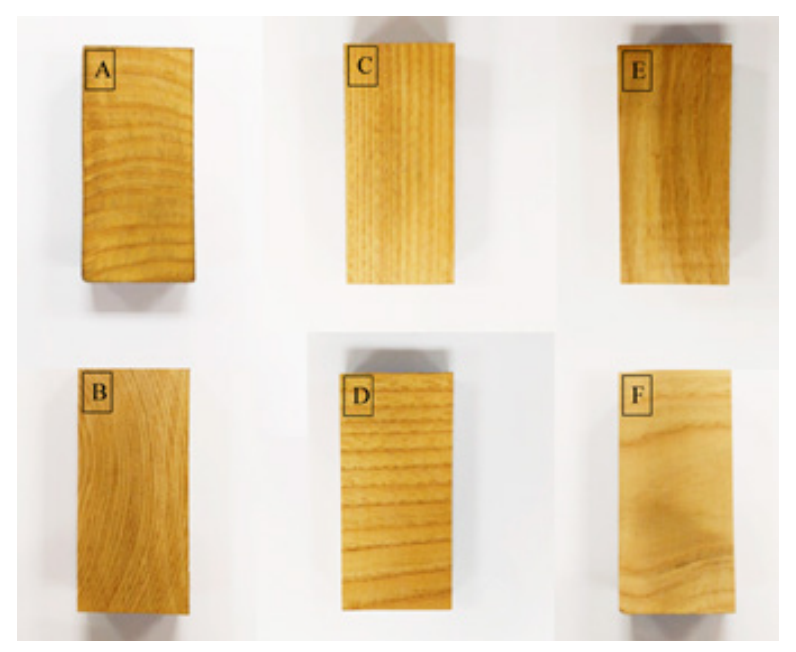

Fig. 6. Examples of surfaces tested following planes and friction directions.

found to be adequate to record the stabilization of the frictional force required to define $\mu_{k}$. According to the ASTM G-115-10 (2018) Standard, the surfaces must reflect the roughness of the timber components under study. Hence, the specimens to be tested must have surfaces that have not gone through either a polishing or roughening process. For this reason, specimens were prepared using a circular saw with a disc intended for use with timber. At the same time, timber orthotropy results in different roughnesses in different planes. In this work, all of the planes were established by the orthotropy of the wood, the different possibilities of the sliding directions were also studied. The cases studied here and the indication of some application to contact surfaces are shown in Figs 5 and 6:

- The transverse surface or section, perpendicular to the grain, with two sliding directions; for example, in frontal encounters in scarf joint or step joints with a low slope:
- Radial sliding direction, following the radius of the annular rings, (A) (Fig. 5).

- Tangential sliding direction, tangential to the annular rings, (B) (Fig. 5).

- Radial surface or section, plane defined by the axis and the radius of the tree trunk:

- Sliding direction parallel to the grain; for example, in the radial surfaces of scarf joints or in step joints with a low slope (C) (Fig. 5).

- Sliding direction perpendicular to the grain; for example, the friction between planks that constitute stresslaminated decks, (D) (Fig. 5).

- Tangential surface or section, tangent to the annular rings:

- Sliding direction parallel to the grain; for example, in the tangential surfaces of scarf joints or in step joints with a slight slope, (E) (Fig. 5).

- Sliding direction perpendicular to the grain; for example, the friction between planks that constitute stresslaminated decks, (F) (Fig. 5).

It should be noted that, in some cases, the sliding direction may not be perfect across all of the specimen surface. This occurs, for example, in case B of Fig. 6, where the slip occurs in the tangential direction to the growth rings but their natural curvature means that the direction cannot be totally tangential at the ends of the specimen surface. This may be viewed as a representation of the reality of the sawn timber, where a direction may be predominant but not necessarily constant and uniform across the whole surface.

Specimens were conditioned at $20 \pm 2^{\circ} \mathrm{C}$ and $65 \pm 5 \%$ humidity, according to CEN EN 408:2011+A1 2012 (2012), which corresponds to an approximately $12 \%$ moisture content (MC) (USDA Forest Products Laboratory, 2010) at the time of testing. This MC corresponds to service class I, timber protected from damp conditions (CEN EN 1995$1-1: 2016,2016)$, which coincides with the conditions that 


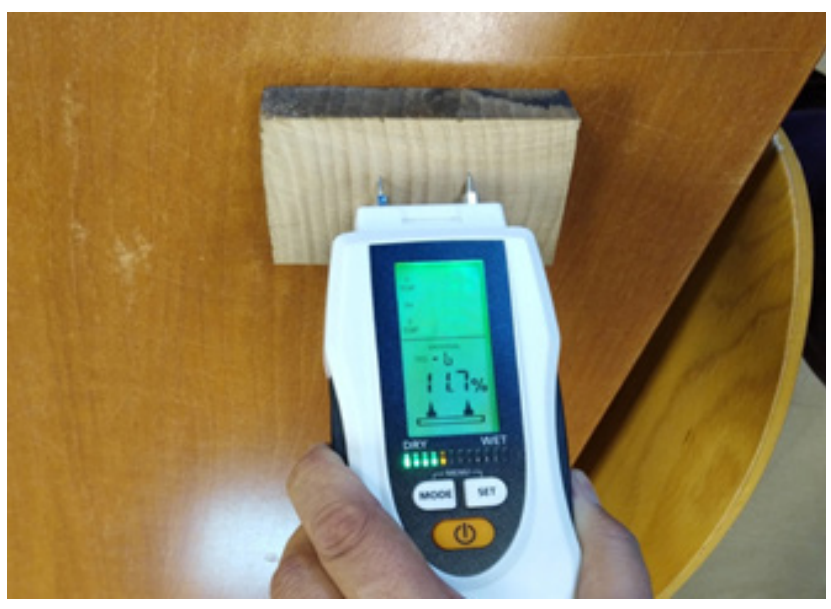

Fig. 7. Moisture content control before test.

predominate in many members and joints where friction takes place. This is in agreement with the recommendation established at the ASTM G-115-10 (2018) Standard, which suggests the consideration of similar conditions to those in use. The specimen moisture content values were checked before each test was conducted (Fig. 7).

The number of tests performed was approximately 25 in each direction. However, in some cases, this figure could not be reached when determining some of the coefficients because its graphic behaviour was not consistent with that of most specimens. Therefore, in a number of tests it was not possible to determine the values of the static or the kinetic friction coefficients, this issue will be discussed in detail.

\section{RESULTS}

In order to determine the evolution of friction in both the static and kinetic stages, the variation of the friction coefficient vs. the displacement was represented graphi- cally in each of the tests carried out according to the cases previously explained. This allowed for the determination of both the static and kinetic coefficients of friction.

The friction between the transverse surfaces and radial sliding direction was studied. Figure 8 shows the graphs with the variation of the friction coefficient versus the displacement registered. Only representative samples of the whole set of data are displayed, thus avoiding any overlap between graphs in order to improve the visualization of the behaviour of the friction coefficient. It may be observed that the graphs reproduce the behaviour of Fig. 2, an initial region, which corresponds to a static situation, and a subsequent region corresponding to a dynamic situation.

In the static region, a linear increase in the friction force occurs until the point or peak of "imminent displacement" already mentioned as a theoretical construct is reached. Once this point is reached, a generally pronounced inflection curve occurs. That maximum constitutes the static friction coefficient, $\mu_{s}$, which may be obtained through the use of Eq. (1). From the different tests conducted, only three did not present this high breakaway force or peak of the corresponding coefficient. Hence, it was considered that they did not precisely represent the behaviour of the material in that aspect and therefore they were discarded with regard to obtaining $\mu_{s}$.

It may be observed that the maximum value appears once a small displacement takes place, which corresponds to an inclination at the beginning of the graph, in most cases lower than $1 \mathrm{~mm}$ and not noted during the test. Although theoretically the two halves of the specimens should not slide with respect to each other before overcoming the "imminent displacement point"; this may be due to, as explained by Aira et al. (2014), a slight slippage as a result of the rupture of micro-weldings between contact faces and because of minor shear deformation in the two halves of the specimen. This slight inclination at the beginning of the graph is considered to be a typical behaviour in the ASTM G-115-10 (2018) Standard.

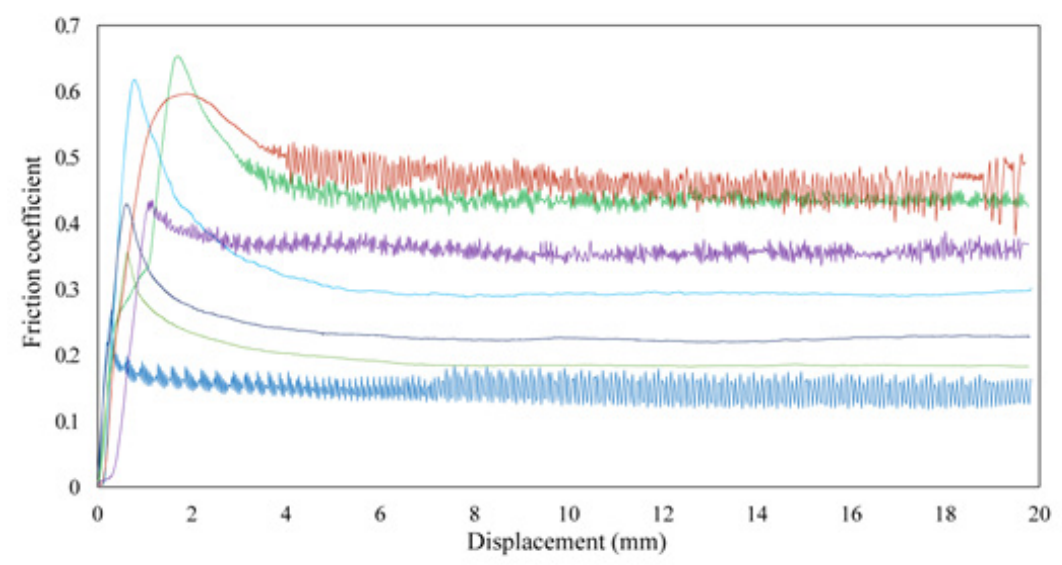

Fig. 8. Friction coefficients vs. displacement for (a) transverse surfaces and radial sliding direction. Only representative samples of the whole set of data are displayed. 
Table 3. Coefficients of friction between transverse surfaces and radial sliding direction

\begin{tabular}{lcccccccccccccccc}
\hline Specimen & 1 & 2 & 3 & 4 & 5 & 6 & 7 & 8 & 9 & 10 & 11 & 12 & 13 & 14 & 15 \\
\hline$\mu_{s}$ & 0.61 & 0.46 & 0.27 & 0.50 & 0.53 & 0.65 & 0.35 & 0.24 & 0.27 & 0.51 & - & 0.32 & 0.65 & 0.49 & - \\
$\mu_{k}$ & 0.29 & 0.34 & 0.21 & 0.33 & 0.48 & 0.44 & - & - & 0.14 & 0.40 & 0.30 & 0.17 & 0.28 & 0.37 & 0.35 \\
Specimen & 16 & 17 & 18 & 19 & 20 & 21 & 22 & 23 & 24 & 25 & 26 & 27 & Average (CoV\%) \\
$\mu_{s}$ & 0.22 & 0.53 & 0.4 & 0.55 & 0.19 & 0.60 & 0.52 & 0.43 & - & 0.64 & 0.36 & 0.43 & $0.45(31.5)$ \\
$\mu_{k}$ & 0.17 & 0.44 & 0.33 & 0.39 & 0.17 & 0.45 & 0.39 & 0.36 & 0.43 & 0.45 & 0.18 & 0.22 & $0.32(32.6)$ \\
\hline
\end{tabular}

Once the maximum value has been exceeded, a decrease in the horizontal load occurs and the magnitude of the coefficient of friction stabilizes around a steady value. For most of the tests conducted, a slight stick-slip behaviour was observed during this dynamic stage. Nevertheless, the small value of this variation allows for the correct identification of the value of the kinetic coefficient $\mu_{\mathrm{k}}$ versus the value of $\mu_{s}$. Also, it was observed that, in most cases, the relative displacement experienced by the specimens was adequate for the stabilization of the friction force, allowing for the estimation of $\mu_{k}$. Table 3 shows both the static and the kinetic coefficients of friction obtained between transverse surfaces and radial sliding direction. In the cases where it was not possible to identify any of the coefficients, no values were taken and therefore the corresponding boxes in Table 3 do not show any numerical value for $\mu_{s}$ and $\mu_{k}$. The mean value obtained for $\mu_{s}$ was 0.45 with a $31.5 \%$ coefficient of variation $(\mathrm{CoV})$. Tests in which no horizontal asymptote occurred were discarded. A kinetic friction coefficient $\mu_{k}$ of 0.32 was obtained with a $32.6 \% \mathrm{CoV}$. The ratio $\mu_{k} / \mu_{s}$ was 0.72 , which means that friction decreases by almost $30 \%$ in the dynamic region.

With respect to the friction between the transverse surfaces and tangential sliding direction, Fig. 9 shows the graphs providing the variation of the coefficient of friction versus displacement. Again, only the representative samples of the data are displayed. The static and the kinetic regions are readily identified and the value of the static friction coefficient may be determined. Although the stickslip effect was again observed in the dynamic region, the stabilization of the values of the friction coefficient allow for the identification of the coefficient of kinetic friction at this stage. Table 4 shows both the coefficients of static and kinetic friction. The mean value obtained for $\mu_{s}$ was 0.39 with a $36.8 \% \mathrm{CoV}$, whereas for $\mu_{k}$, the mean value was 0.25 with a $39.9 \% \mathrm{CoV}$. The ratio $\mu_{k} / \mu_{s}$ was 0.63 in this case.

Also, the friction between the radial surfaces and the sliding direction parallel to the grain was analysed. Figure 10 provides the graphs of representative samples showing the evolution of the coefficient of friction versus the displacement. The behaviour is similar to that observed in previous cases, allowing for the identification in most cases of a clear peak for the static coefficient of friction and a horizontal asymptote for the kinetic one. Table 5 shows both the static and the kinetic friction coefficients obtained. The mean value obtained for $\mu_{s}$ was 0.41 with a $30.7 \% \mathrm{CoV}$, whereas for $\mu_{k}$, the mean value was 0.31 with a $35.2 \% \mathrm{CoV}$. The ratio $\mu_{k} /$ $\mu_{s}$ was 0.76 , which means that a friction decrease of about $24 \%$ took place in the kinetic region.

The results obtained of the friction occurring between the radial surfaces and the sliding direction perpendicular to the grain are shown in Fig. 11. This figure shows the graphs of representative samples with the variation of the coefficient of friction versus the displacement registered.

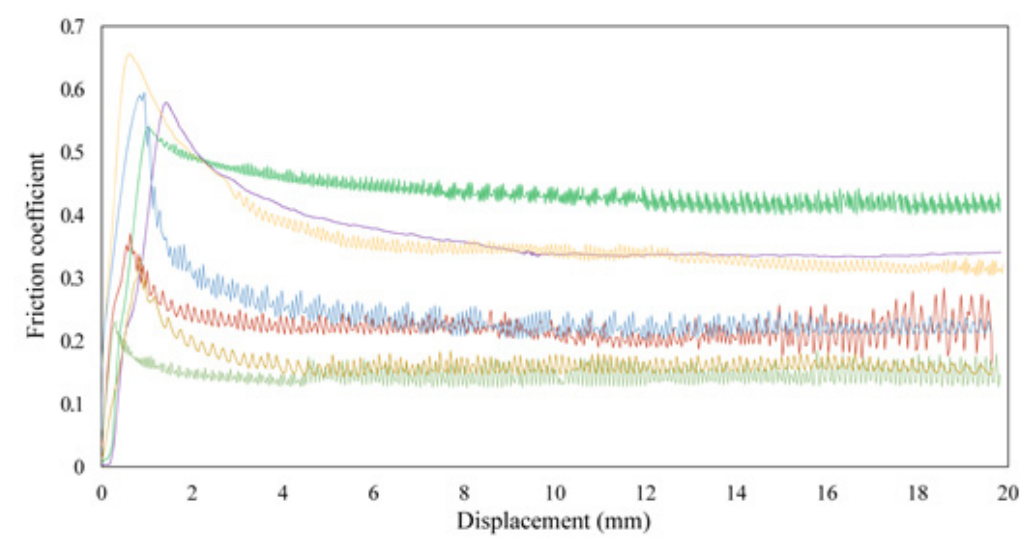

Fig. 9. Friction coefficients $v s$. displacement for (c) transverse surfaces and tangential sliding direction. Explanation as on Fig. 8. 
Table 4. Coefficients for friction between transverse cut surfaces and tangential direction of sliding

\begin{tabular}{|c|c|c|c|c|c|c|c|c|c|c|c|c|c|c|}
\hline Specimen & 1 & 2 & 3 & 4 & 5 & 6 & 7 & 8 & 9 & 10 & 11 & 12 & 13 & 14 \\
\hline$\mu_{s}$ & 0.26 & 0.42 & 0.29 & 0.57 & 0.38 & 0.45 & 0.52 & 0.54 & 0.45 & 0.66 & 0.37 & 0.23 & 0.42 & 0.21 \\
\hline$\mu_{k}$ & 0.23 & 0.35 & 0.26 & 0.34 & 0.3 & 0.36 & 0.45 & 0.42 & 0.4 & 0.31 & 0.22 & 0.14 & 0.21 & 0.18 \\
\hline Specimen & 15 & 16 & 17 & 18 & 19 & 20 & 21 & 22 & 23 & 24 & 25 & \multicolumn{3}{|c|}{ Average (CoV\%) } \\
\hline$\mu_{s}$ & 0.18 & 0.33 & 0.41 & 0.73 & 0.35 & 0.59 & 0.33 & 0.27 & 0.36 & 0.2 & 0.32 & \multicolumn{3}{|c|}{$0.39(36.8)$} \\
\hline$\mu_{k}$ & 0.13 & 0.16 & - & 0.29 & 0.15 & 0.22 & 0.19 & 0.14 & 0.16 & 0.14 & 0.18 & \multicolumn{3}{|c|}{$0.25(39.8)$} \\
\hline
\end{tabular}

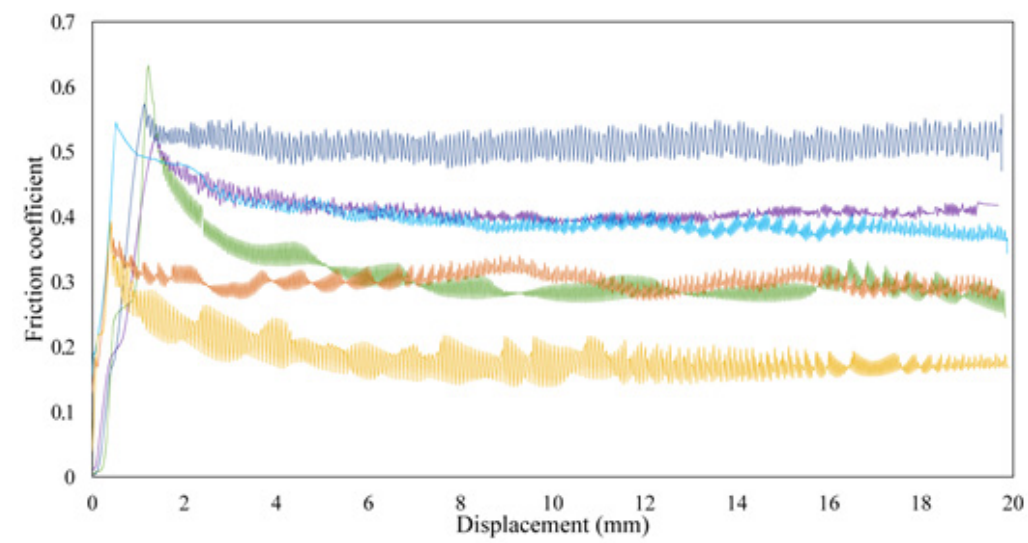

Fig. 10. Friction coefficients $v s$. displacement for (e) radial surfaces and sliding direction parallel to the grain. Explanation as on Fig. 8.

Table 5. Coefficients for friction between radial surfaces and direction of sliding parallel to the fibre

\begin{tabular}{lcccccccccccccc}
\hline Specimen & 1 & 2 & 3 & 4 & 5 & 6 & 7 & 8 & 9 & 10 & 11 & 12 & 13 & 14 \\
\hline$\mu_{s}$ & 0.52 & 0.38 & - & - & 0.27 & 0.31 & - & 0.57 & 0.63 & 0.23 & 0.28 & 0.4 & 0.32 & 0.4 \\
$\mu_{k}$ & 0.41 & 0.32 & 0.3 & 0.46 & 0.15 & - & 0.42 & 0.41 & 0.29 & 0.17 & 0.21 & 0.33 & 0.16 & 0.2 \\
Specimen & 15 & 16 & 17 & 18 & 19 & 20 & 21 & 22 & 23 & 24 & 25 & Average (CoV\%) \\
$\mu_{s}$ & 0.4 & 0.56 & - & 0.54 & 0.4 & 0.28 & 0.39 & 0.29 & 0.28 & 0.59 & 0.55 & $0.41(30.7)$ \\
$\mu_{k}$ & 0.35 & 0.3 & 0.44 & 0.42 & 0.17 & - & 0.3 & 0.25 & 0.18 & 0.51 & 0.39 & $0.31(35.2)$ \\
\hline
\end{tabular}

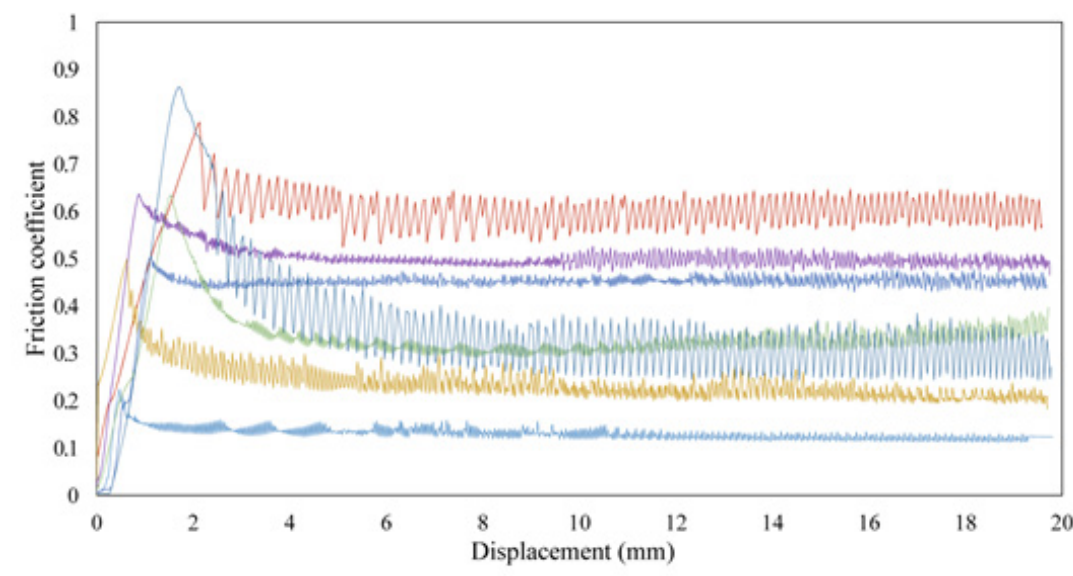

Fig. 11. Friction coefficients $v s$. displacement for (a) radial surfaces and sliding direction perpendicular to the grain. Explanation as on Fig. 8 . 
Table 6. Coefficients for friction between radial surfaces and sliding direction perpendicular to the fibre

\begin{tabular}{lccccccccccccccc}
\hline Specimen & 1 & 2 & 3 & 4 & 5 & 6 & 7 & 8 & 9 & 10 & 11 & 12 & 13 & 14 & 15 \\
\hline$\mu_{s}$ & 0.79 & 0.44 & 0.5 & 0.53 & 0.25 & 0.22 & 0.49 & 0.38 & 0.57 & 0.51 & 0.43 & 0.47 & 0.24 & 0.33 & 0.64 \\
$\mu_{k}$ & 0.6 & 0.4 & 0.22 & 0.51 & 0.17 & 0.16 & 0.32 & 0.43 & 0.3 & 0.46 & - & 0.32 & 0.17 & 0.17 & 0.49 \\
Specimen & 16 & 17 & 18 & 19 & 20 & 21 & 22 & 23 & 24 & 25 & 26 & 27 & 28 & $\begin{array}{c}\text { Average } \\
(\mathrm{CoV} \%)\end{array}$ \\
$\mu_{s}$ & 0.38 & 0.64 & 0.36 & 0.45 & 0.81 & 0.71 & 0.68 & 0.67 & 0.56 & 0.55 & 0.57 & 0.64 & 0.86 & $0.52(32.3)$ \\
$\mu_{k}$ & 0.24 & 0.33 & 0.28 & 0.24 & 0.61 & 0.5 & 0.5 & 0.4 & 0.22 & 0.21 & 0.3 & - & 0.3 & $0.34(40.2)$ \\
\hline
\end{tabular}

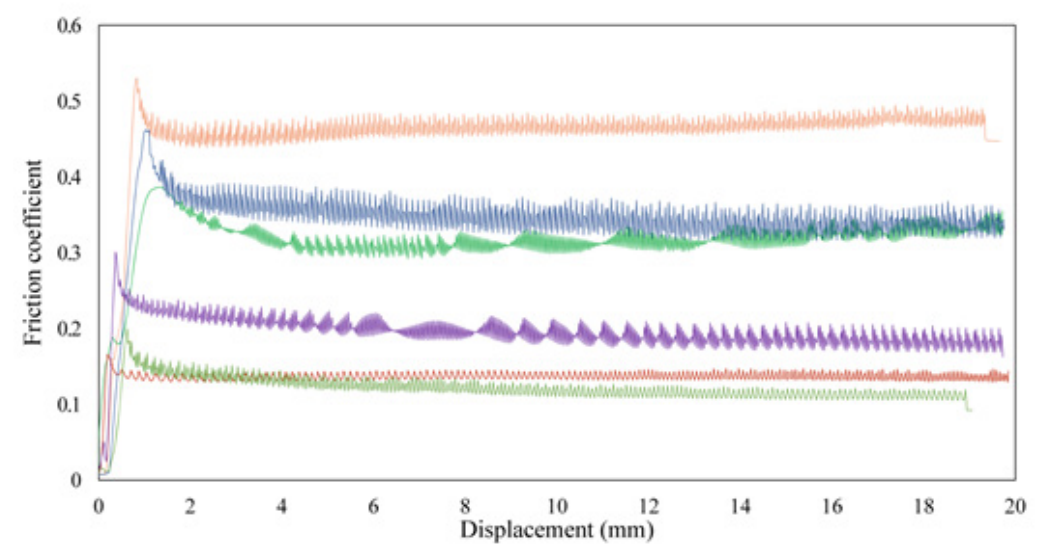

Fig. 12. Friction coefficients vs. displacement for (c) tangential surfaces and sliding direction parallel to the grain. Explanation as on Fig. 8 .

Table 7. Coefficients for friction between tangential surfaces and sliding direction parallel to the fibre

\begin{tabular}{cccccccccccccccc}
\hline Specimen & 1 & 2 & 3 & 4 & 5 & 6 & 7 & 8 & 9 & 10 & 11 & 12 & 13 \\
\hline$\mu_{s}$ & 0.36 & 0.24 & 0.39 & - & 0.35 & 0.48 & 0.26 & 0.34 & 0.5 & 0.34 & 0.53 & 0.35 & 0.17 \\
$\mu_{k}$ & 0.18 & 0.22 & 0.32 & 0.25 & 0.30 & - & 0.20 & 0.31 & 0.43 & 0.19 & 0.47 & 0.27 & 0.13 \\
Specimen & 14 & 15 & 16 & 17 & 18 & 19 & 20 & 21 & 22 & 23 & 24 & $\begin{array}{c}\text { Average } \\
(\mathrm{CoV} \%)\end{array}$ \\
$\mu_{s}$ & 0.20 & 0.30 & 0.47 & 0.40 & 0.43 & 0.35 & - & 0.46 & 0.28 & 0.17 & 0.51 & $0.36(30.3)$ \\
$\mu_{k}$ & 0.11 & 0.18 & 0.43 & 0.27 & 0.40 & 0.35 & 0.37 & 0.34 & 0.24 & 0.14 & 0.45 & $0.28(38.7)$ \\
\hline
\end{tabular}

The behaviour was similar to that observed in previous cases, allowing for the identification in most cases of the static and kinetic coefficient of friction. Table 6 shows the coefficients obtained; in this case, the mean value obtained for $\mu_{s}$ was 0.52 with a $32.3 \% \mathrm{CoV}$, whereas for $\mu_{k}$, the mean value obtained was 0.34 with a $40.2 \% \mathrm{CoV}$. The ratio $\mu_{k} / \mu_{s}$ was 0.65 .

As for the friction between tangential surfaces and the sliding direction parallel to the grain, Fig. 12 shows the graphs with the variation of the coefficient of friction versus the displacement reached. Table 7 shows both the static and kinetic coefficients of friction obtained. In this case, the average value obtained for $\mu_{s}$ was 0.36 with a $30.3 \% \mathrm{CoV}$, whereas for $\mu_{\mathrm{k}}$, an average value of 0.28 was obtained with a $38.7 \% \mathrm{CoV}$. The ratio of $\mu_{k} / \mu_{s}$ was 0.78 in this case.
Finally, the friction between the tangential surfaces and the sliding direction perpendicular to the grain was studied. Fig. 13 shows the representative graphs with the variation of the coefficient of friction vs. the displacement reached. Table 8 shows both the static and the kinetic coefficients of friction obtained for tangential surfaces and the sliding direction perpendicular to the grain. In this case, the average value obtained for $\mu_{s}$ was 0.55 with a $23.9 \% \mathrm{CoV}$, whereas for $\mu_{k}$, an average value of 0.39 was obtained, with a $34.9 \% \mathrm{CoV}$. In this case, the ratio of $\mu_{k} / \mu_{s}$ was 0.71 .

\section{DISCUSSION}

Table 9 summarizes the results provided, showing the mean values obtained for both the static and the kinetic coefficients of friction depending on the orientation of 


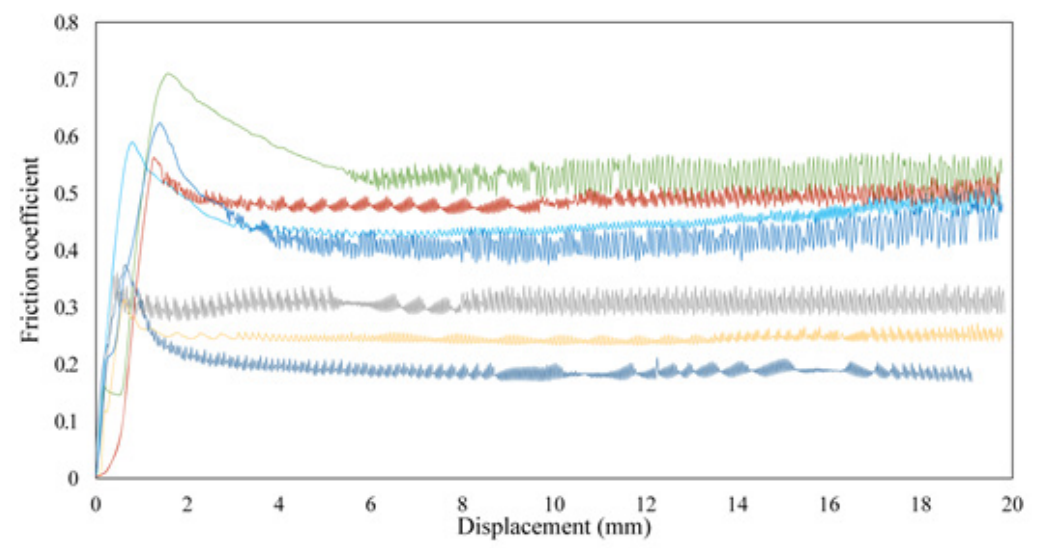

Fig. 13. Friction coefficients vs. displacement for (e) tangential surfaces and sliding direction perpendicular to the grain. Explanation as on Fig. 8.

Table 8. Friction coefficients between tangential surfaces and sliding direction perpendicular to the grain

\begin{tabular}{ccccccccccccccc}
\hline Specimen & 1 & 2 & 3 & 4 & 5 & 6 & 7 & 8 & 9 & 10 & 11 & 12 & 13 & 14 \\
\hline$\mu_{s}$ & 0.64 & 0.57 & - & 0.59 & 0.33 & 0.54 & 0.71 & 0.36 & 0.44 & 0.63 & 0.71 & 0.45 & 0.72 & 0.59 \\
$\mu_{k}$ & 0.51 & - & 0.17 & 0.52 & 0.17 & 0.49 & 0.51 & 0.32 & 0.41 & 0.45 & 0.52 & 0.20 & 0.55 & 0.42 \\
Specimen & 15 & 16 & 17 & 18 & 19 & 20 & 21 & 22 & 23 & 24 & 25 & 26 & $\begin{array}{c}\text { Average } \\
(\text { CoV\%) }\end{array}$ \\
$\mu_{s}$ & 0.31 & 0.59 & 0.56 & 0.69 & 0.51 & 0.62 & 0.57 & 0.47 & 0.76 & 0.41 & - & 0.37 & $0.55(23.9)$ \\
$\mu_{k}$ & 0.25 & - & 0.5 & 0.48 & 0.49 & 0.44 & 0.49 & 0.43 & - & 0.2 & 0.25 & 0.18 & $0.39(34.9)$ \\
\hline
\end{tabular}

the surfaces and the sliding directions. From Table 9, the mean values obtained without considering the different planes and friction directions were 0.45 for the static friction coefficient $\left(\mu_{s}\right)$ and 0.32 for the kinetic one $\left(\mu_{k}\right)$. They had coefficients of variation $(\mathrm{CoV})$ of $16.8 \%$ for $\mu_{s}$ and $15.4 \%$ for $\mu_{k}$ with regard to the different directions considered. This confirms that the orientation of the surfaces and shear directions must be taken into account when studying friction in chestnut timber. These mean values were within the range of the general values provided by other authors for timber: 0.25 to 0.50 for the static coefficient and a value slightly higher than 0.20 was reported in the literature for the kinetic coefficient (Kollmann, 1959; Serway and Jewett, 2013), but a value within the range 0.3 to 0.5 was suggested for the kinetic coefficient by the Wood Handbook (USDA Forest Products Laboratory, 2010) for a 12\% moisture content. McKenzie and Karpovich (1968) provided a static coefficient of friction of 0.6 and a kinetic one of 0.45 without specifying either directions or species, which would result in higher values than those obtained here. In addition, the values provided here were within the range suggested by Koch et al. (2013) for dry spruce: 0.3 to 0.8 for $\mu_{s}$ and 0.3 to 0.6 for $\mu_{k}$.
A global mean value of 0.71 was obtained for the ratio of $\mu_{k} / \mu_{s}$. This value was close to $25 \%$ lower with respect to kinetic friction compared to the static one, generally indicated for sliding between solids (Keller et al., 1993; Tipler, 2008; Serway and Jewett, 2013; Young and Freedman, 2016). Figure 14 shows the correlation between $\mu_{s}$ and $\mu_{k}$ for all of the tests carried out, and the mean values for each type of friction. When relating the mean values of $\mu_{s}$ and $\mu_{k}$, a good correlation may be observed, thereby obtaining an acceptable coefficient $\mathrm{R}^{2}=0.83$ and indicating that once the static coefficient of friction is obtained, the kinetic one could be estimated by using the expression: $\mu_{k}=0.59 \mu_{s}+$ 0.05 .

When analysing the different sections considered, it may be appreciated that the highest values for both the static and the kinetic friction are reached for radial and tangential surfaces at the sliding direction perpendicular to the grain. The values obtained for these surfaces ranged between $16-23 \%$ and between $8-24 \%$ higher than the mean values obtained for $\mu_{\mathrm{s}}$ and $\mu_{\mathrm{k}}$ respectively. These results show the effect of the wood fibres when a slip perpendicular to the grain takes place, even in surfaces parallel to it resulting from a movement of rolling between fibres. Moreover, the lowest values obtained corresponded to the surface sliding tangential to 


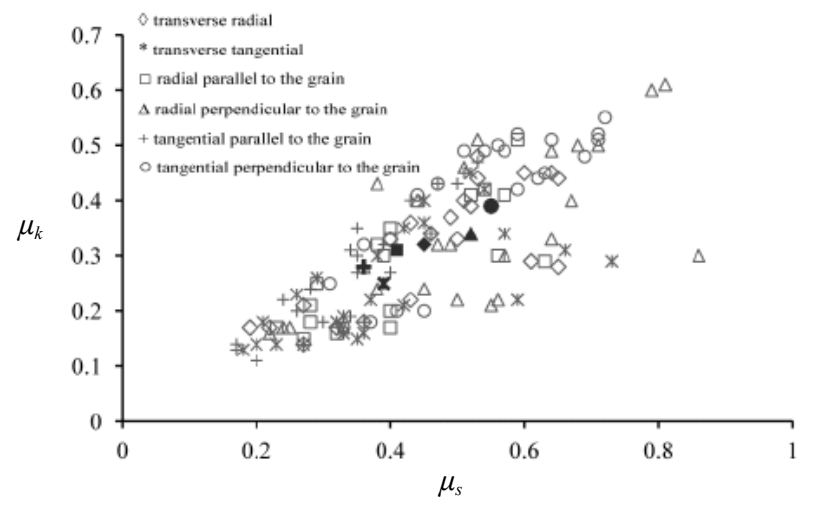

Fig. 14. Relationships between the mean values of $\mu_{k}$ and $\mu_{s}$ according to the different surfaces and friction directions considered, indicating the mean values for each case (black symbols).

the direction parallel to the fibre. These values were $19 \%$ and $11 \%$ lower than the mean ones obtained for $\mu_{s}$ and $\mu_{k}$, respectively, which shows the effect of the lower intersection with the growth rings and fibres. In the case of $\mu_{k}$, the low value obtained for the transverse surfaces and tangential sliding direction should also be highlighted. In all these cases the close relationship between $\mu_{s}$ and $\mu_{k}$ can be appreciated since the maximum and minimum static values also corresponded with the kinetic ones.

For each surface orientation, the values of the $\mathrm{CoV}$ obtained in this work were within the common values for mechanical tests on timber in which, even after a quality classification, $\mathrm{CoV}$ values of over $25 \%$ are frequent (Argüelles et al., 2013). However, in some cases, for the kinetic coefficients of friction, the $\mathrm{CoV}$ values were high (in one case it reached a value of $40.2 \%$ ); these high values have also been reported by other authors given the natural variability of wood (Aira et al., 2014).

As for the relationship with the values provided by other authors for the different surfaces considered, the comparisons discussed below may be made. In any case, the lack of studies for some of them should be noted, since in many cases several directions are grouped together, even though they have been taken into account individually in this work. (i) For surfaces resulting from transversal cuts to the grain, mean values of 0.42 for $\mu_{s}$ and 0.29 for $\mu_{k}$ were obtained. These values were similar to those reported by Crespo et al. (2011) for laminated timber of Picea abies L. Karst, 0.467 for $\mu_{s}$ and 0.310 for $\mu_{k}$ in the intermediate sliding direction radial-tangential, being slightly closer to these values than those obtained in this work for radial sliding ( 0.45 for $\mu_{s}$ and 0.32 for $\mu_{k}$ ). Aira et al. (2014) provided values of 0.24 for $\mu_{s}$ and 0.17 for $\mu_{k}$ for Scots pine when studying transversal surfaces and the tangential sliding direction, lower than those obtained in this work for that same direction $\left(0.39\right.$ for $\mu_{s}$ and 0.25 for $\left.\mu_{k}\right)$. While for cuts perpendicular to the fibre and oak timber, Kollmann (1959) provided values of 0.54 and 0.34 for $\mu_{s}$ and $\mu_{k}$ (Table 2), which are similar to those values obtained here. Koch et al. (2013) provided values of 0.45 for $\mu_{s}$ for surfaces perpendicular to the fibre rubbing against surfaces at $45^{\circ}$ for spruce, which may be related to a certain affinity with the surfaces that this point refers to, thus observing that the value provided herein is similar. Finally, for softwoods in general and moisture contents lower or equal than $12 \%$, with friction perpendicular to the fibre, the Standard Eurocode 5-Part 2: Bridges (CEN EN 1995-2:2016, 2016) states a design value for the static coefficient of 0.30 (Table 1), which may be considered as a conservative value.

(ii) For sliding parallel to the grain, mean values of 0.41 and 0.31 were obtained for $\mu_{s}$ and $\mu_{k}$ respectively for the radial surface, and the values of these coefficients were $0.36\left(\mu_{s}\right)$ and $0.28\left(\mu_{k}\right)$ for the tangential surface. These values may be compared with the value of the static coefficient provided by the Standard Eurocode 5-Part 2: Bridges (CEN EN 1995-2:2016, 2016) for conifer timber, 0.23 for $\mu_{s}$, which is a low value, even lower than those obtained here, it may be considered a conservative value. A higher value was provided by Kollmann (1959) for oak timber and surfaces parallel to the grain: 0.62 for $\mu_{s}$ and 0.48 for $\mu_{k}$ (Table 2). Finally, Aira et al. (2014) obtained lower values than those provided here for radial surfaces and sliding in the direction of the fibre for Scots pine timber $\left(0.12\right.$ for $\mu_{s}$ and 0.08 for $\left.\mu_{k}\right)$. He indicated that the fibre inclination in this surface as well as other aspects generate lower homogeneity than that obtained for transversal surfaces, and therefore a higher variability in the results obtained.

Table 9. Values for the static and kinetic friction coefficients. Mean values (CoV\%)

\begin{tabular}{llccc}
\hline Surface & Sliding direction & $\mu_{s}$ & $\mu_{k}$ & $\mu_{k} / \mu_{s}$ \\
\hline \multirow{2}{*}{ Transverse } & radial & $0.45(31.5)$ & $0.32(32.6)$ & 0.72 \\
& tangential & $0.39(36.8)$ & $0.25(39.8)$ & 0.63 \\
\multirow{3}{*}{ Radial } & parallel to the grain & $0.41(30.7)$ & $0.31(35.2)$ & 0.76 \\
& perpendicular to the grain & $0.52(32.3)$ & $0.34(40.2)$ & 0.65 \\
\multirow{2}{*}{ Tangential } & parallel to the grain & $0.36(30.3)$ & $0.28(38.7)$ & 0.78 \\
& perpendicular to the grain & $0.55(23.9)$ & $0.39(34.9)$ & 0.71 \\
\hline
\end{tabular}


(iii) In the case of slipping perpendicular to the grain, and surfaces parallel to it, either radial or tangential, similar values were obtained: 0.52 for $\mu_{s}$ and 0.34 for $\mu_{k}$ for radial surfaces, and 0.55 for $\mu_{s}$ and 0.39 for $\mu_{k}$ for tangential surfaces. The only close reference to this type of friction may be found in the tests carried out by Soilán et al. (2011) with glulam of picea for obtaining the friction coefficients in dovetail joint flanks, giving a coefficient of static friction of 0.2 and a kinetic coefficient of 0.27 . These results are for an intermediate between the radial and the tangential surfaces parallel to the grain, due to the configuration of the laminates of the glulam, in the tail's flanks, with sliding occurring perpendicular to it (although in Soilan's test, they rub perpendicular to the grain surfaces), thus resulting in values slightly lower than those obtained in this work. On the other hand, the value provided by the Eurocode 5-Part 2: Bridges (CEN EN 1995-2:2016, 2016) for static friction perpendicular to the grain $\left(\mu_{s}=0.30\right)$ may be also considered for these surfaces, since it was provided for friction between planks in stress-laminated decks. Again, its value is conservative for application to structural calculations.

The results obtained in this work are within the range of values reported in the literature. However, the comparison carried out between them was limited since those values are for other species, or in general for timber, or they do not provide information about the directions studied. In any case, the authors considered that it was interesting to provide the results of such comparisons. In addition, the variability which is added to the results by the different test methods used by several authors has to be taken into account.

\section{CONCLUSIONS}

1. It has been observed that the methodology developed herein to obtain the coefficients of friction for timber using a modified direct shear device is reliable. It allows the static coefficient of friction to be obtained. The displacement in the device is also adequate for characterizing the kinetic coefficient of friction. The results using this apparatus were compared to work conducted by different authors but because of the variation in each test apparatus, the results were difficult to compare. Because of this a standardized method must be developed. The method described here may contribute to this goal since the direct shear device is a machine commonly available in material laboratories worldwide.

2. Nearly all of the tests conducted presented a typical friction behaviour. They reached a maximum value for the friction coefficient, coincident with the imminent sliding point, thus corresponding to the static friction coefficient, and a later kinetic region where the coefficient of friction remains constant, this corresponds to the kinetic coefficient. Therefore, both coefficients were easily determined for different surfaces and sliding directions.
3. Mean values, without considering the different directions, of 0.45 for the static friction coefficient and 0.32 for the kinetic coefficient of friction were obtained. These values were within the interval of general values provided by several authors for timber. The highest values obtained for both the static and the kinetic friction coefficient were reached for radial and tangential surfaces with the direction of sliding perpendicular to the fibre, followed by those obtained for surfaces transverse to the grain. This showed the important effect that the fibre has on friction when sliding perpendicular to the grain takes place. The lowest values obtained corresponded to tangential surfaces with a sliding direction parallel to the grain. This also showed the effect of the lack, or slight, intersection with the annual rings.

4. In all cases, static friction coefficient $\left(\mu_{s}\right)$ was higher than kinetic friction coefficient $\left(\mu_{k}\right)$. Based on these tests, for chestnut timber, the kinetic coefficient of friction, once the static one is known, may be determined according to the ratio $\mu_{k}=0.59 \mu_{s}+0.05$.

Conflict of interests: The authors declare that they have no conflict of interest.

\section{REFERENCES}

Aira J.R., Arriaga F., Iniguez-Gonzalez G., and Crespo J., 2014. Static and kinetic friction coefficients of Scots pine (Pinus sylvestris L.), parallel and perpendicular to grain direction. Mater. Constr., 64(315). https://doi.org/10.3989/mc.2014.03913

Aira J.R., Íñiguez-González G., Guaita M., and Arriaga F., 2016. Load carrying capacity of halved and tabled tenoned timber scarf joint. Mater. Struct., 49(12), 5343-5355. https://doi.org/10.1617/s11527-016-0864-y

Argüelles R., Arriaga F., Esteban M., Iñíguez G., and Argüelles Bustillo R., 2013. Timber Structures. Basis for Calculation (in Spanish). AITIM. Technical Research Association of the Wood and Cork Industries, Madrid, Spain.

ASTM G115-10(2018), 2018. Standard Guide for Measuring and Reporting Friction Coefficients. Am. Soc. Testing Materials, West Conshohocken, PA, USA. https://doi.org/10.1520/G0115-10R18

Bejo L., Lang E.M., and Fodor T., 2000. Friction coefficients of wood-based structural composites. For. Prod. J., 50(3), 39-43.

CEN EN 1995-1-1:2016, 2016. Eurocode 5: Design of timber structures - Part. 1.1 General. Common rules and rules for buildings. European Committee for Standardisation, Brussels, Belgium. https://doi.org/10.3403/03174906u

CEN EN 1995-2:2016, 2016. Eurocode 5: Design of timber structures - Part. 2. Bridges. European Committee for Standardisation, Brussels, Belgium.

CEN EN 408:2011+A1 2012, 2012. Timber structures - Structural timber and glued laminated timber - Determination of some physical and mechanical properties. European Committee for Standardization, Brussels, Belgium.

https://doi.org/10.3403/00547175u

Crespo J., Regueira R., Soilan A., Díez M.R., and Guaita M., 2011. Methodology to determine the coefficients of both 
static and dynamic friction apply to different species of wood. In: Proc. 1st Ibero-Latin American Congr. Wood in Construction. CIMAD 11, June 7-9, Coimbra, Portugal. https://doi.org/978-989-96461-2-4

Keller F.J., Gettys W.E., and Skove M.J., 1993. Physics, classical and modern. McGraw-Hill, New York, USA.

Koch H., Eisenhut L., and Seim W., 2013. Multi-mode failure of form-fitting timber connections - Experimental and numerical studies on the tapered tenon joint. Eng. Struct., 48, 727-738. https://doi.org/10.1016/j.engstruct.2012.12.002

Kollmann F., 1959. Wood technology and its applications. Forestry Institute for Research, Experiences and Wood Service. Department of Agriculture, Madrid, Spain.

McKenzie W.M. and Karpovich H., 1968. The frictional behaviour of wood. Wood Sci. Technol., 2(2), 139-152. https://doi.org/10.1007/BF00394962

Molenda M., Stasiak M., Moya M., Ramirez A., Horabik J., and Ayuga F., 2006. Testing mechanical properties of food powders in two laboratories - degree of consistency of results. Int. Agrophysics, 20, 37-45.

Moya M., Aguado P.J., and Ayuga F., 2013. Mechanical properties of some granular agricultural materials used in silo design. Int. Agrophys, 27(2), 181-193. https://doi.org/10.2478/v10247-012-0084-9

Serway R.A. and Jewett J.W., 2013. Physics for Scientists and Engineers with Modern Physics. Cengage Learning, New York, USA.

Soilán A., Arriaga F., Baño V., Crespo J., and Guaita M., 2011. Analysis of the behavior of the dovetail connection by numerical simulation with the finite element method., in: Proceeding of the 1er Ibero-Latin American Congress on Wood in Construction. CIMAD 11, June 7-9, Coimbra, Portugal. https://doi.org/978-989-96461-2-4

Tipler P.A., 2008. Physics for scientists and engineers. W.H. Freeman-Worth, 1999 4th ed., New York, USA.

USDA Forest Products Laboratory, 2010. Wood handbook: wood as an engineering material. General Technical Report FPLGTR-190. Madison, WI, USA. https://doi.org/10.2737/FPL-GTR-190

Villar J.R., Guaita M., Vidal P., and Argüelles R., 2008. Numerical simulation of framed joints in sawn-timber roof trusses. Spanish J. Agric. Res., 6(4), 508-520. https://doi.org/10.5424/sjar/2008064-345

Villar J.R., Guaita M., Vidal P., and Arriaga F., 2007. Analysis of the stress state at the cogging joint in timber structures. Biosyst. Eng., 96(1), 79-90. https://doi.org/10.1016/j.biosystemseng.2006.09.009

Villar-García J.R., Crespo J., Moya M., and Guaita M., 2018. Experimental and numerical studies of the stress state at the reverse step joint in heavy timber trusses. Mater. Struct., 51(1), 17. https://doi.org/10.1617/s11527-018-1144-9

Villar-García J.R., Vidal-López P., Crespo J., and Guaita M., 2019. Analysis of the stress state at the double-step joint in heavy timber structures. Mater. Constr., 69(335), e196. https://doi.org/10.3989/mc.2019.00319

Young H.D. and Freedman R.A., 2016. University Physics with Modern Physics. Pearson Education, San Francisco, USA. 